\title{
In Vivo Assessment of Acute UVB Responses in Normal and Xeroderma Pigmentosum (XP-C) Skin-Humanized Mouse Models
}

\author{
Marta García, ${ }^{\star \dagger}$ Sara Llames, ${ }^{\dagger \neq}$ Eva García, ${ }^{\dagger \ddagger}$ \\ Alvaro Meana, ${ }^{\dagger \ddagger}$ Natividad Cuadrado, ${ }^{\dagger}$ \\ Mar Recasens, ${ }^{\S}$ Susana Puig, " Eduardo Nagore,"

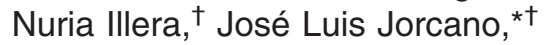 \\ Marcela Del Rio, ${ }^{\star \dagger}$ and Fernando Larcher* \\ From the Cutaneous Regenerative Medicine Unit, ${ }^{*}$ Epithelial \\ Biomedicine Division, CIEMAT, and CIBERER-U714, ${ }^{\dagger}$ Madrid; \\ Centro Comunitario de Sangre y Tejidos de Asturias, ${ }^{\ddagger}$ Puig \\ Research Centre, Barcelona; the Melanoma Unit, ${ }^{\Phi}$ Hospital \\ Clinic and CIBERER-U726, Barcelona; and the Dermatology \\ Department," Instituto Valenciano de Oncologia, Valencia, Spain
}

In vivo studies of UVB effects on human skin are precluded by ethical and technical arguments on volunteers and inconceivable in cancer-prone patients such as those affected with Xeroderma Pigmentosum (XP). Establishing reliable models to address mechanistic and therapeutic matters thus remains a challenge. Here we have used the skin-humanized mouse system that circumvents most current model constraints. We assessed the UVB radiation effects including the sequential changes after acute exposure with respect to timing, dosage, and the relationship between dose and degree-sort of epidermal alteration. On Caucasian-derived regenerated skins, UVB irradiation $\left(800 \mathrm{~J} / \mathrm{m}^{2}\right)$ induced DNA damage (cyclobutane pyrimidine dimers) and 553 expression in exposed keratinocytes. Epidermal disorganization was observed at higher doses. In contrast, in African descent-derived regenerated skins, physiological hyperpigmentation prevented tissue alterations and DNA photolesions. The acute UVB effects seen in Caucasian-derived engrafted skins were also blocked by a physical sunscreen, demonstrating the suitability of the system for photoprotection studies. We also report the establishment of a photosensitive model through the transplantation of XP-C patient cells as part of a bioengineered skin. The inability of XP-C engrafted skin to remove DNA damaged cells was confirmed in vivo. Both the normal and XP-C ver- sions of the skin-humanized mice proved proficient models to assess UVB-mediated DNA repair responses and provide a strong platform to test novel therapeutic strategies. (Am J Pathol 2010, 177:865-872; DOI: 10.2353/ajpath.2010.091096)

Excessive exposure to solar UV radiation causes severe acute damages to the skin, including erythema, sunburn, immunosupression, and also long-term effects such as photoaging and skin cancer. ${ }^{1-5}$ UV effects are greatly exacerbated in patients suffering from Xeroderma Pigmentosum (XP), an autosomal and recessive disorder characterized by a severe deficiency in the most versatile DNA-repair mechanism in charge of the removal of bulky DNA adducts including UV-induced cyclobutane pyrimidine dimmers (CPDs) and pyrimidine pyrimidone photoproducts (6-4PPs). The study of the in vivo biological effects of UV irradiation on human volunteers is restricted for ethical and technical reasons, namely frequency of sampling and size of the skin biopsies and homogeneity of samples. Different animal models have been used to study the in vivo effects of UV light on the skin. However, all of them present significant limitations. One of the most widespread, the mouse model, weakens as a model to study UV effects due to the differences between mouse and human skin in terms of epidermal thickness/architecture and DNA repair abilities. Mouse skin is far thinner and hairier than human skin and in addition, global nucleotide excision repair (G-NER), the most important DNA repair mechanism acting in humans, appears less relevant in mouse skin. ${ }^{6,7}$ To circumvent these restrictions, models based on grafting human skin biopsies on to

Supported in part by grants P-BIO-0306-2006 from Comunidad de Madrid, PSE-010000-2008-7 from MICINN, and PI081054 from ISCIII (MICINN) (F.L.) and grants SAF 2007-61019 (M.D.R.) and INTRA/07/726,2 from CIBERER (S.P.).

Accepted for publication April 19, 2010.

Address reprint requests to Fernando Larcher, Ph.D., Cutaneous Regenerative Medicine Unit, Epithelial Biomedicine Division, CIEMATCIBERER-U714, Av Complutense 22, 28040 Madrid, Spain. E-mail: Fernando.larcher@ciemat.es. 
immunodeficient mice have been developed to study UVB-induced effects. ${ }^{6,8}$ These systems are a useful way to combine the human native skin organization with the corresponding preservation of its typical human DNA lesion repair mechanisms. In addition, human skin biopsies grafted to immunodeficient mice have also been shown to be of value to study UVB-induced carcinogenesis. ${ }^{9-11}$ However, these approaches are again limited for ethical and technical constraints. Under such an experimental setting, each skin biopsy from a volunteer is used to generate a single grafted mouse. Thus, the possibility to perform a large study in a homogenous population is hampered. It is worth mentioning that neither the former nor other kind of humanized in vivo models have been reported for XP patients. An alternative to whole skin biopsy grafting is to achieve human skin regeneration on mice on engraftment of tissue-engineered skin equivalents. This approach offers the unique opportunity to generate animals engrafted with identical skin samples. In fact, only one skin biopsy donation may suffice to generate a big surface of bioengineered skin constructs, enabling in turn the production of a large number of skin-humanized mice. ${ }^{12}$ A new skin-humanized mouse model based on the grafting of a skin equivalent, previously used in clinics for permanent skin regeneration in burn patients, ${ }^{13,14}$ was recently shown to be a suitable model for the study of physiological processes such as wound healing in a human context. ${ }^{15,16}$ Moreover, the platform allows trustworthy and efficient modeling and gene therapy of inherited skin diseases such as different forms of Epidermolysis Bullosa. ${ }^{17-19}$ These results prompted us to test the performance of our model to study the effects of UV under normal skin conditions and to seek a new UV hypersensitive model.

In the present work we characterized the in vivo biological acute effects of UV irradiation on the skin-humanized mouse model by assessing the histopathological changes elicited at different doses. Changes including epidermal architecture alterations, increased keratinocyte proliferation, and DNA damage were analyzed. In addition, we report the development and characterization of a photosensitive XP-C skin-humanized mouse model, a suitable platform to gain new insight on UVB biological effects and DNA repair-dependent pathogenesis and to test novel therapeutic approaches for this rare cancerprone inherited disease.

\section{Materials and Methods}

\section{Normal and XP-C Skin Samples}

All human skin samples were donated with informed consent and with the approval of the Hospital Clinic de Barcelona ethics committee. Normal human foreskin samples were obtained from phimosis/circumcision surgery from Caucasian and black (African-descent) 0 - to 1-year-old donors. Skin biopsies of XP-C were obtained from two different patients from nonlesional skin areas. Determination of XP complementation group was carried out in keratinocytes by retroviral infection with different vectors expressing wild-type XP cDNAs as described. ${ }^{20}$

\section{Primary Cultures of Normal and XP-C Human Keratinocytes and Fibroblasts}

Human keratinocytes and dermal fibroblasts were obtained from skin biopsies of donors by enzymatic digestion. ${ }^{20}$ Primary keratinocytes were cultured on a feeder layer of lethally irradiated (X-ray; 50 Gy) 3T3-J2 cells (a gift from Dr J. Garlick Tufts University) as previously described. ${ }^{22,23}$ The keratinocyte seeding media was a 3:1 mixture of Dulbecco's modified Eagle medium (DMEM) (GIBCOBRL, Barcelona, Spain) and HAM'S F12 containing $10 \%$ fetal calf serum replacement (Fetal Clone II, Hyclone-Lonza), $0.1 \mathrm{nmol} / \mathrm{L}$ cholera toxin, $2 \mathrm{nmol} / \mathrm{L}$ T3, 5 $\mu \mathrm{g}$ per $\mathrm{ml}$ insulin, and $0.4 \mu \mathrm{g}$ per $\mathrm{ml}$ hydrocortisone. Primary fibroblasts, obtained through collagenase digestion after tripsinization of biopsies, were cultured in DMEM containing $10 \% \mathrm{FCS}$. Cells were cultured at $37^{\circ} \mathrm{C}$ in a humid atmosphere containing $5 \% \mathrm{CO}_{2}$. The culture medium was changed every 2 days.

\section{Bioengineered Skin Preparation and Grafting to Immunodeficient Mice}

The bioengineered human skin equivalent is based on the use of a fibrin matrix containing live fibroblasts (either normal or XP-C) as a dermal component. ${ }^{22,23}$ Briefly, $3 \mathrm{ml}$ of the fibrinogen (from cryoprecipitates) was added to 12 $\mathrm{ml}$ of DMEM with $10 \%$ FCS containing $5 \times 105$ dermal fibroblasts and 500 IU of bovine aprotinin (Trasylol; Bayer, West Haven, CT). Immediately afterward, $1 \mathrm{ml}$ of $0.025 \mathrm{mmol} / \mathrm{L} \mathrm{CaCl} 2$ (Sigma, St. Louis, MO) with $11 \mathrm{IU}$ of bovine thrombin (Sigma) was added. Finally, the mixture was placed on a six-well culture plate (Transwell; Costar, Cambridge, MA) and allowed to solidify at $37^{\circ} \mathrm{C}$ for 2 hours. Normal or XP-C keratinocytes were then seeded and grown submerged up to keratinocyte confluence. Immunodeficient nu/nu female mice (NMRI strain; Elevage-Janvier, France) were aseptically cleansed and grafted as previously described elsewhere. ${ }^{14,22}$ Briefly, full thickness 12-mm circular wounds were then created on the dorsum of the mice. Bioengineered equivalents were placed orthotopically on the wound and covered with devitalized mouse skin. Mice were housed for the duration of the experiment at the CIEMAT Laboratory Animals Facility (Spanish registration number 28079-21 A) in pathogen-free conditions using individually ventilated type II cages (25 air changes per hour) and 10 KGy-irradiated soft wood pellets as bedding.

\section{UV Irradiations of Skin-Humanized Mice}

The UVB source was two fluorescent sunlamps (Philips, UVB TL20W/12RS), with peak at $312 \mathrm{~nm}$ (UV in the B range, 290 to $320 \mathrm{~nm}$ ). The wavelength was carefully determined with a spectroradiometer (UVX radiometer, UVP Inc., Upland, CA). The UVB irradiance was $2.0 \mathrm{mw}$ / 
$\mathrm{cm}^{2}$. UVB irradiation (single dose) was performed on stable engrafted mice (9-12 weeks post grafting). Biological efficient UV dose (BED) was determined through irradiation of Caucasian-derived cell engrafted mice at different doses ranging from 400 to $1200 \mathrm{~J} / \mathrm{m}^{2}$. 6,24 In our setting, the UV dose giving rise to sunburn cells 24 hours after irradiation (1 BED) was established at $800 \mathrm{~J} / \mathrm{m}^{2}$. For photoprotection analysis, human engrafted skin was topically treated with sunscreen cream (SPF-90, ISDIN, Barcelona) $\left(2 \mathrm{mg} / \mathrm{cm}^{2}\right)$. Animals were irradiated 30 minutes after sunscreen application.

\section{Histopathological Analysis of UV Exposed Human Skin}

Control or UV-irradiated mice were sacrificed by $\mathrm{CO}_{2}$ inhalation. Tissue samples (2 $\mathrm{mm}$ biopsy punches (STIEFEL LAB, Madrid, Spain) of each engrafted mouse were collected at different time points after UV exposure. Skin samples were harvested and fixed in 3.7\% buffered formaldehyde. After fixation the skin biopsy was embedded in paraffin. Serial 4- $\mu \mathrm{m}$ cross-sections were obtained. The UV-irradiated human skin (normal and XP-C) was analyzed by histological and immunohistochemical techniques. Paraffin sections were dewaxed and rehydrated in water solutions containing decreasing percentages of ethanol. To determine tissue architecture and detect sunburn cells, sections were stained with hematoxylin-eosin (Gill 2 Hematoxylin and Eosin Y alcoholic; Thermo Shandon, Cheshire, UK) following a standard procedure. The human origin of the grafts was determined by immunostaining using a human specific antiinvolucrin monoclonal antibody (clone SY-5, Sigma) that decorated the regenerated human skin but not the mouse skin, as previously described. ${ }^{14,15,22}$ The human epidermal-associated proliferative marker keratin K6 was labeled with LHK6B monoclonal antibody (NeoMarkers. CA). Immunofluorescent detection was performed using keratin K5 (Covance), and Proliferating Cell Nuclear Antigen (PCNA) (Thermo Scientific) antibodies.

To evaluate the DNA damage induced by UVB we used mouse monoclonal antibodies against CPDs at final dilution 1:1000 (H3 clone 4F6, Afitech) and p53 (clone DO-7, Dako) at final dilution 1:20. To assess epidermal cell proliferation before and after UV irradiation, grafted mice were IP injected with $20 \mathrm{mg}$ per $\mathrm{kg}$ of BrdU 1 hour before euthanasia. BrdU immunodetection was performed using specific antibodies against BrdU (Roche, IN) at a 1:50 dilution. Immunoperoxidase staining was developed using the Vectastain ABC kit (Vector, Burlingame, CA).

\section{Statistical Analysis}

A Student's t-test was applied to compare the means of samples using Microsoft Excel (Microsoft, Redwood, CA). Differences were considered statistically significant when $P<0.05$.
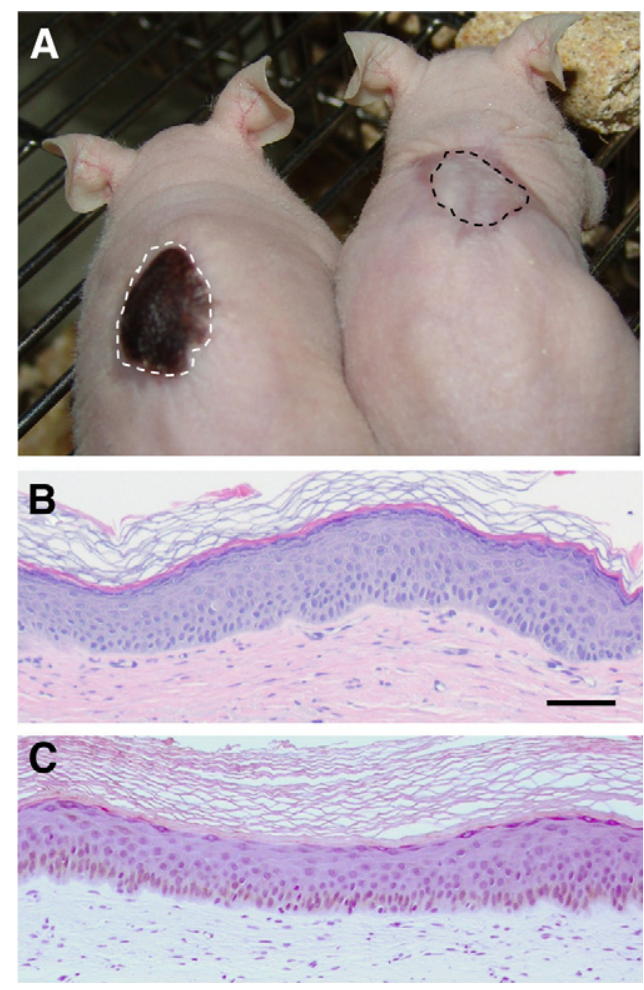

Figure 1. Macro and microscopic appearance of Caucasian and African descent-derived regenerated skins. A: Phenotypical appearance of mice engrafted with skin regenerated either from Caucasian (right) or African descent-derived (left) keratinocytes (including melanocytes) and fibroblasts. Grafts are delimited by dotted lines. B: Histological appearance (H\&E staining) of Caucasian donor-derived regenerated skin 12 weeks after grafting. C: Histological appearance (H\&E staining) of African descent-derived regenerated skin 12 weeks after grafting. Note the brownish color due to (unstained) melanin in the basal cell compartment. Scale bar $=100 \mu \mathrm{m}$.

\section{Results}

\section{Acute UVB Responses on Normal Skin-Humanized Mice}

The performance of the skin-humanized mice to acute UVB irradiation effects was assessed on mice engrafted after transplantation of bioengineered skin equivalents containing normal human epidermal cells (including resident melanocytes) from either Caucasian or African donors of identical age and location source (ie, foreskin) (Figure 1A). Despite the large macro and microscopic pigmentation differences, the histological features of both human engrafted skins were very similar in terms of thickness, differentiated strata number, and stratum corneum maturation (Figure 1, B and C).

A UVB BED, measured as the minimal dose inducing histological sunburn cells (SBC), ${ }^{6,23}$ was first determined in dose response experiments performed on nonpigmented skin-humanized mice. In our setting, a $800 \mathrm{~J} / \mathrm{m}^{2}$ dose of UVB was established as 1 BED (see Materials and Methods).

Twenty four hours after exposure to a sub BED dose (0.5 BED) of UVB, a marked differential response between Caucasian and African donor-derived skins was observed. In fact, nonpigmented skin reacted by thickening and concomitant induction of the hyperprolifera- 


\section{Caucasian}
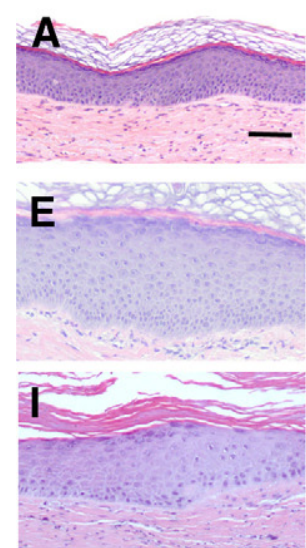
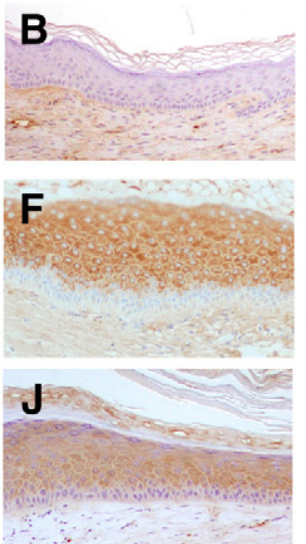

African
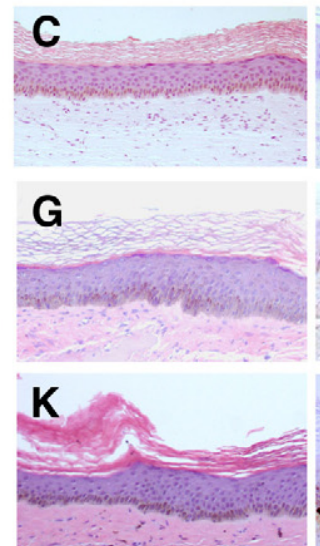
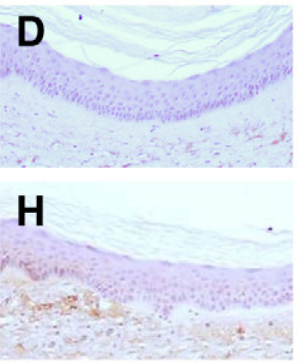

$\mathbf{L}$

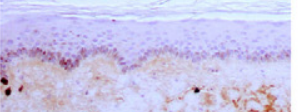

M

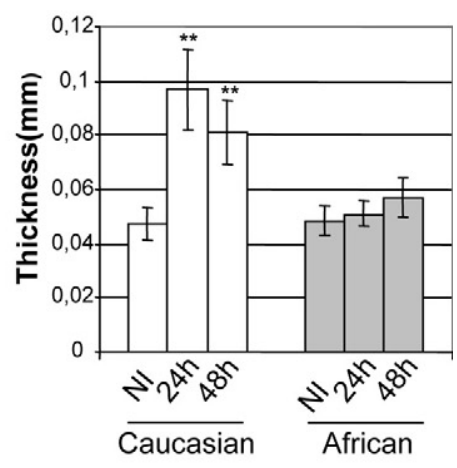

Figure 2. Histopathological changes after low-dose (0.5 BED) UVB irradiation in skin humanized mice A-D: Histological sections of regenerated human skins taken immediately before 0.5 BED of UVB irradiation. A and C: Hematoxylin-eosin (H\&E) staining. B and D: Immunoperoxidase staining of keratin K6. E-H: Histological sections of regenerated human skins taken 24 hours after 0.5 BED. E and G: H\&E staining. F and H: Keratin K6 immunoperoxidase staining. Note the acanthosis (thickening) and K6-positive staining of Caucasian-derived skin (E and $\mathbf{F}$, respectively). I-L: Histological sections of regenerated human skin taken 48 hours after 0.5 BED. I and K: H\&E staining. J and L: Keratin K6 immunoperoxidase staining. Panels A, B, E, F, I, and $\mathbf{J}$ correspond to Caucasian-derived regenerated skin. Panels $\mathbf{C}, \mathbf{D}, \mathbf{G}, \mathbf{H}, \mathbf{K}$, and $\mathbf{L}$ correspond to African descent-derived regenerated skins. Scale bar $=100 \mu \mathrm{m}$. $\mathbf{M}$ : Quantitative analysis of epidermal thickness at 24 and 48 hours after irradiation. Open bars indicate Caucasian-derived skins; shaded bars, African-derived skins. Scale bar $=100 \mu \mathrm{m}$. *** $P<0.01$.

tion-associated keratin K6 (Figure 2, E and F) as compared with nonirradiated skin (Figure 2, A, B, and M). By contrast, pigmented skin appeared to be completely inert both in terms of acanthotic and keratin $\mathrm{K} 6$ induction responses (Figure 2, C, D, G, H, and $\mathrm{M}$ ). It is worth noting that, as predicted, a 0.5 BED dose was unable to induce either SBC or CPD in the nuclei of exposed keratinocytes (see below). Forty-eight hours after 0.5 BED irradiation, epidermal acanthosis was still present in Caucasian-derived skin but quantitative data showed a slight, although not statistically significant, decline. Persistence of epidermal thickening 48 hours after irradiation was accompanied by concomitant maintenance of keratin K6 expression (Figure 2, I and J). In contrast to Caucasian-derived skin, hyperpigmented skin remained unaffected (Figure 2, $\mathrm{K}$ and $\mathrm{L}$ ).

The protective action of high melanin content in regenerated skins was evaluated 24 hours after a supra BED (4 BED). In contrast to that found at 0.5 BED (Figure 2) or 1 BED (Figure 3A) doses, Caucasian donor-derived regenerated skins underwent an important tissue damage characterized by keratinocyte spongiosis, abundant SBC, and epidermal blistering (Figure $3 C)$. By contrast, African descent donor-derived skin irradiated with 4 BED preserved its normal epidermal architecture despite the presence of SBC (Figure 3D) as compared with the unaffected, 1 BED-irradiated skin (Figure 3B). This result indicates that the high pigmentation confers, semiquantitatively, a fourfold protection from the acute effects of UVB. In other words, in our setting, a 4 BED for Caucasian donor-derived skin represents nearly 1 BED for pigmented skin. Noteworthy, skin architecture was preserved in pigmented skin even at a 8 BED (data not shown).

To assess the suitability of the system for photoprotection studies, we also tested the efficacy of a strong physical sunscreen (SPF 90) on Caucasian donor-derived skin engrafted mice. Topical application of the product
30 minutes before a 4 BED dose completely blocked the effects of UVB including the hyperplastic nongenotoxic ones seen at a sub BED (0.5 BED) dose (Figure 3E and data not shown).
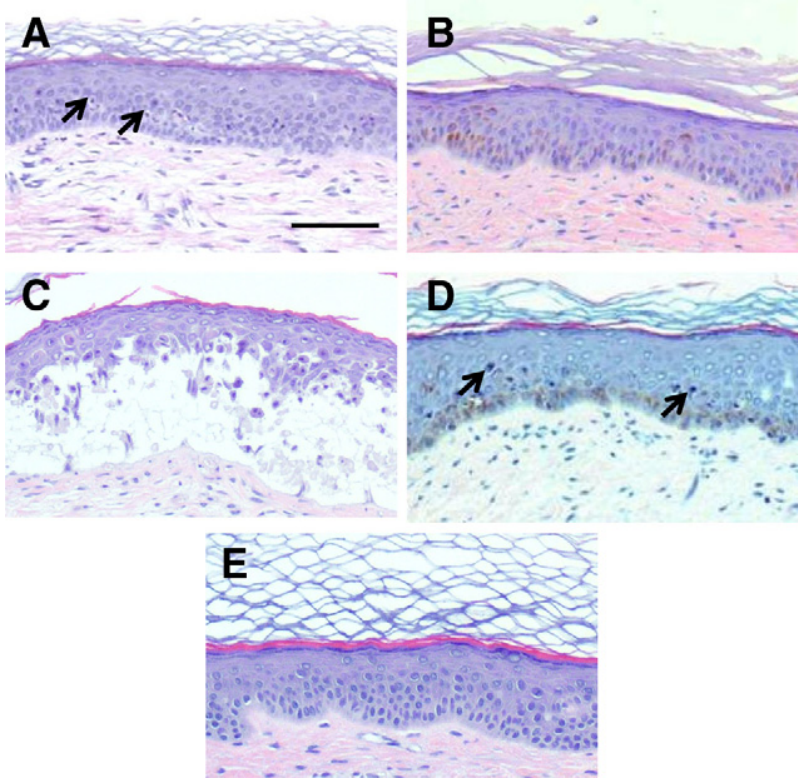

Figure 3. Histopathological changes after high-dose (4 BED) UVB irradiation in Caucasian, African descent-derived, and photoprotected (Caucasian) regenerated skins. A: Histological appearance (H\&E staining) of a representative section of 1 BED irradiated Caucasian-derived skin 24 hours after irradiation. B: Histological appearance of a representative section of $1 \mathrm{BED}$ irradiated African descent-derived skin 24 hours after irradiation. C: Histological appearance (H\&E staining) of a representative section of 4 BEDirradiated Caucasian-derived skin 24 hours after irradiation. Note the disruption of the epidermal architecture. D: Histological appearance (H\&E staining) of a representative section of 4 BED-irradiated African descent-derived skin 24 hours after irradiation. Arrows indicate sunburn cells. E: Histological appearance (H\&E staining) of a representative section of a photoprotected (SPF 90), UVB-irradiated (4 BED) Caucasian-derived skin 24 hours after irradiation. Note the absence of acanthosis, epidermolysis, and sunburn cells. 

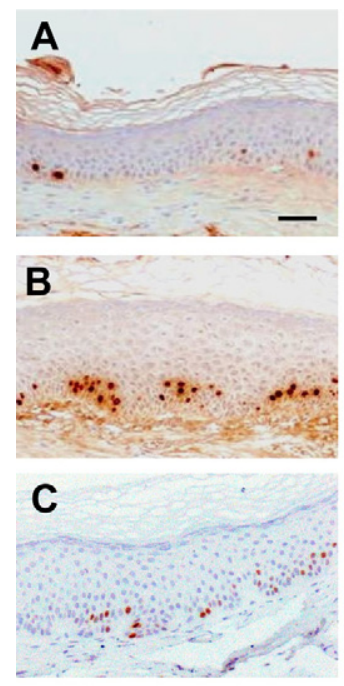

D
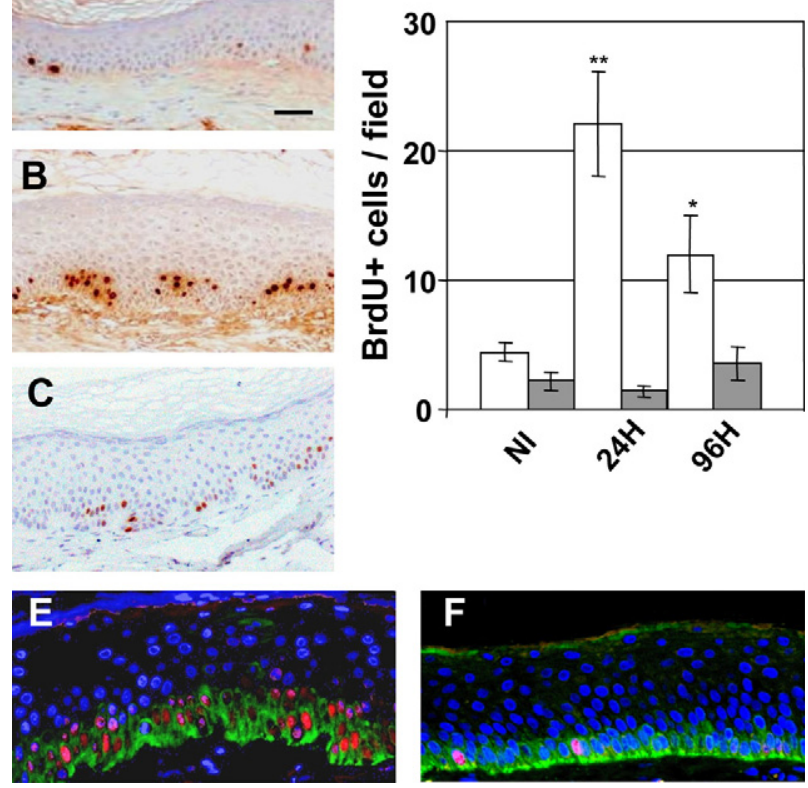

Figure 4. Kinetic analysis of epidermal cell proliferation (BrdU incorporation) after 0.5 BED of UVB. A: BrdU staining of nonirradiated human (Caucasian) regenerated skin. B: BrdU staining of 0.5 BED-irradiated human (Caucasian) regenerated skin 24 hours after irradiation. C: BrdU staining of 0.5 BED-irradiated human (Caucasian) regenerated skin 96 hours after irradiation. Scale bar $=100 \mu \mathrm{m}$. D: Quantitative analysis of BrdU incorporation 24 hours and 96 hours after 0.5 BED irradiation. Open bars: Caucasianderived skins. Shaded bars: African-derived skins. ${ }^{*} P<0.05 ;{ }^{* *} P<0.01$ $\mathbf{E}$ and F: Double immunofluorescence staining for Keratin K5 (green) and PCNA (red) in Caucasian (E) and African (F)-derived skins 24 hours after 0.5 BED irradiation. Nuclei are counterstained with DAPI (blue).

Both the hyperplastic and SBC biological responses have a molecular correlate at the DNA level as an increase of BrdU-labeled nuclei and the induction of single DNA strand breaks measured as CPD, respectively. The kinetics of BrdU changes were analyzed by immunoperoxidase in both Caucasian and African descent donorengrafted skins irradiated with 0.5 BED. As expected, nonirradiated quiescent regenerated human skin showed few BrdU labeled nuclei (Figure 4A). Consistent with the epidermal thickening observed at 0.5 BED in Caucasianderived skins (Figure 2), a fivefold increase of BrdUlabeled nuclei was found $(4.4 \pm 1.5$ versus $22.07 \pm 8.02$ labeled nuclei per field) 24 hours post irradiation (Figure 4B). In contrast, as expected from the absence of acanthosis, African descent donor-derived grafts showed no increase in proliferating cells $(2.2 \pm 1.4$ versus $1.4 \pm 0.8$ labeled nuclei per field). Ninety-six hours after irradiation, the hyperproliferative response in Caucasian donor-derived skin remained high but attenuated respect to that observed at 24 hours $(12 \pm 2.9$ versus $22.07 \pm 1.4$ labeled nuclei per field) (Figure 4C) and maintained at control levels in hyperpigmented grafts (Figure 4D).

To better characterize the proliferating cell population we performed a double immunofluorescence analysis of PCNA and keratin K5, a marker of basal epidermal cells, 24 hours after a 0.5 BED irradiation. Consistent with the BrdU immunoperoxidase data, PCNA nuclear labeling was very robust in Caucasian donor-derived skins but
A

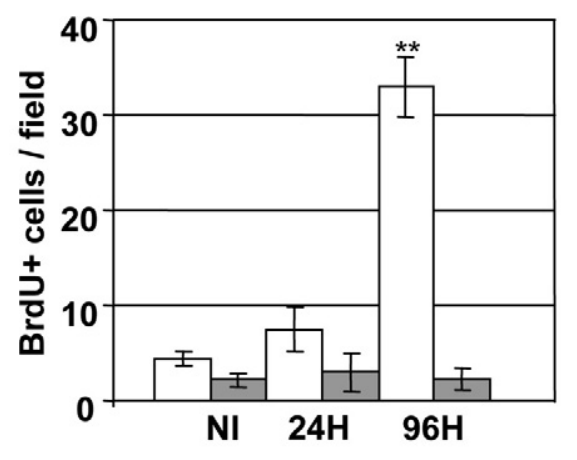

$24 \mathrm{~h}$

$96 \mathrm{~h}$
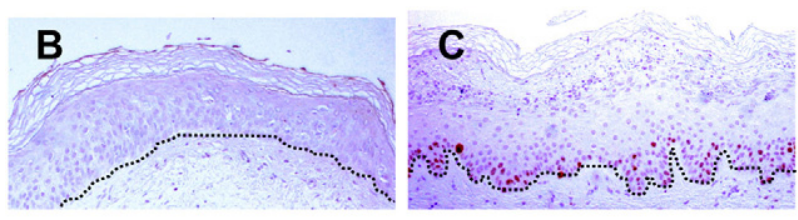

\section{D}
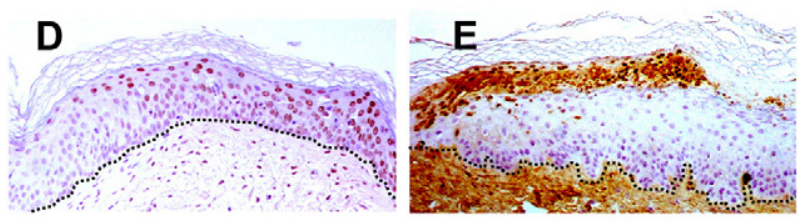

$\mathbf{F}$

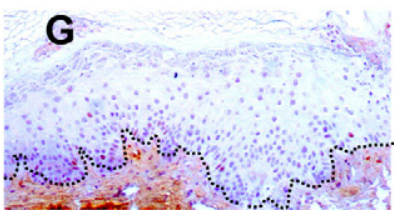

Figure 5. Kinetic analysis of epidermal cell proliferation (BrdU incorporation) and DNA damage (CPD) after 1 BED of UVB. A: Quantitative analysis of BrdU incorporation 24 hours and 96 hours after 1 BED irradiation. Open bars: Caucasian-derived skins. Shaded bars: African-derived skins. ${ }^{* * *} P<0.01$. B: BrdU staining of 1 BED-irradiated human (Caucasian) regenerated skin 24 hours after irradiation. Note the absence of BrdU labeling. C: BrdU staining of 1 BED-irradiated human regenerated skin (Caucasian) 96 hours after irradiation. D: CPD immunostaining of 1 BED-irradiated human regenerated skin 24 hours after irradiation. E: CPD immunostaining of 1 BED-irradiated human regenerated skin (Caucasian) 96 hours after irradiation. Note the strong positive staining of heavily damaged cells in their transit to desquamation. F: p53 immunostaining of 1 BED-irradiated human regenerated skin 24 hours after irradiation. G: p53 immunostaining of 1 BED-irradiated human regenerated skin 96 hours after irradiation. Dotted lines depict the epidermal-dermal border.

comparable to that of unirradiated skin in hyperpigmented grafts. In both graft types, PCNA-positive nuclei colocalized exclusively with K5-labeled cells (Figure 4, E and F). At 0.5 BED no immunoreaction to CPD was detected at any time point indicating that DNA damage was either low or absent. (data not shown).

Analysis performed in Caucasian skin grafts 24 hours after 1 BED irradiation showed a remarkably low BrdU incorporation (Figure 5, A and B). However, 96 hours post irradiation a robust delayed proliferative response and concomitant hyperplasia was observed (Figure 5C). By contrast, in hyperpigmented skins, BrdU incorporation remained at control levels (nonirradiated skin) at both time points (Figure 5A), suggesting that a threshold dose capable to induce the hyperproliferative response, seen at 0.5 BED in Caucasian skins, was not reached. 


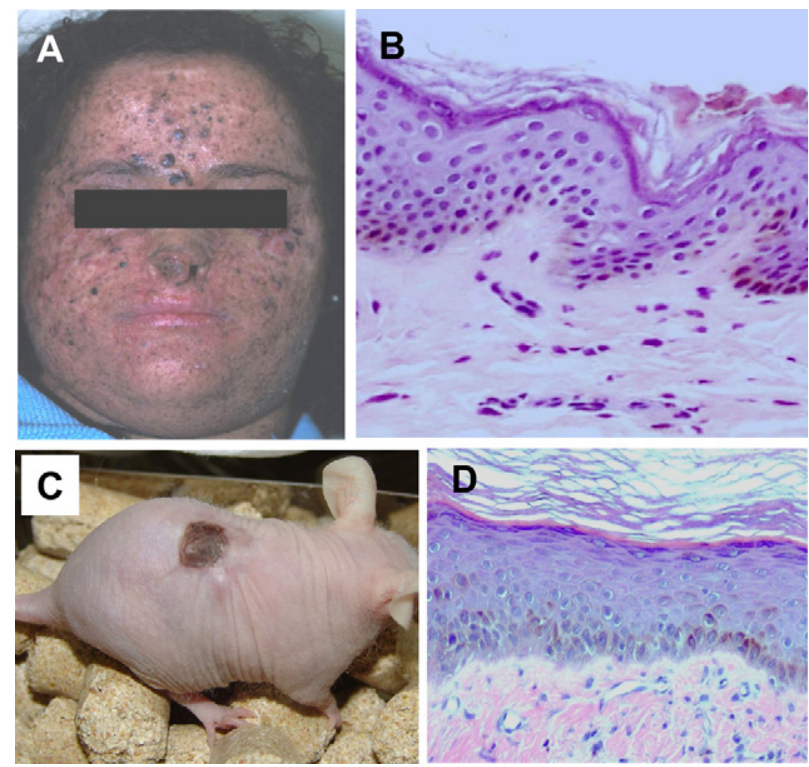

Figure 6. Development of a XP-C skin-humanized mouse model. A: Physical characteristic appearance of one of the XP-C donor patients. B: Histological appearance (H\&E staining) of a section from the patient's skin biopsy used to culture skin cells. C: Appearance of a representative XP-C regenerated skin-engrafted mouse. Note that cells from patient 1 (shown in $\mathbf{A}$ ) give rise to pigmented regenerated skin. D: Histological appearance (H\&E staining) of a section of the regenerated human skin in a mouse engrafted with cells from patient in $\mathbf{A}$

The differential proliferative response between 0.5 and 1 BED doses seen 24 hours after irradiation in Caucasian skins is clearly explained by the fact that at $1 \mathrm{BED}$, almost all epidermal nuclei were labeled for CPD (Figure 5D) and p53 (Figure 5F). In contrast to the extinction of the hyperproliferative response seen 96 hours after a 0.5 $\mathrm{BED}$, a reactive hyperproliferation burst appears to be required, after $1 \mathrm{BED}$, to trigger an accelerated differentiation and desquamation process to rapidly eliminate those heavily DNA-damaged cells. In fact, 96 hours after irradiation CPD-labeled cells were pictured only at a highly suprabasal location in their transit to desquamation (Figure 5E). The fact that neither basal nor early suprabasal cells maintained detectable CPD indicates that efficient DNA repair has occurred within the proliferative cell compartment allowing a proliferative restart (Figure 5C). Consistently, no p53-positive cells were detected at this time point (Figure 5G). These results confirms previous findings obtained in vitro on skin equivalents. ${ }^{25}$

\section{Development of a XP-C Skin-Humanized Mouse Model and Assessment of Acute UVB Responses}

Previous work from our laboratory focused on the development of trustworthy in vivo models for inherited skin diseases to test novel therapeutic approaches including gene therapy. ${ }^{12,17-19}$ Those results prompted us to seek for a humanized in vivo model for the photosensitive and cancer-prone disease XP. To that end, keratinocytes and fibroblasts from nonlesional areas of two different patients (Figure 6A) suffering from XP-C, the most common
$2 \mathrm{~h}$
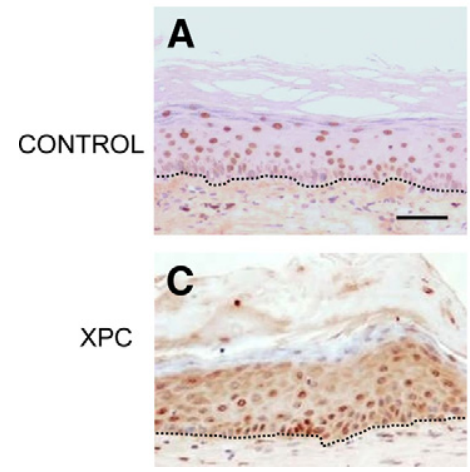

$72 \mathrm{~h}$
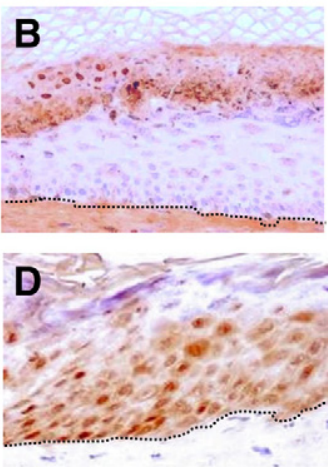

Figure 7. In vivo inability to repair DNA damage (CPD) in XP-C regenerated skin. A: CPD immunostaining of 4 BED-irradiated normal African-derived skin (control) section two hours after irradiation. B: CPD immunostaining of 4 BED-irradiated control skin section 72 hours after irradiation. C: CPD immunostaining of 4 BED-irradiated XP-C regenerated skin section 2 hours after irradiation. D: CPD immunostaining of 4 BED-irradiated XP-C regenerated skin section 72 hours after irradiation. Note the persistence of CPDlabeled cells in all epidermal strata indicating a DNA damage repair defect. Dotted lines depict the epidermal-dermal border. Scale bar $=50 \mu \mathrm{m}$.

form of XP, were cultured, assembled in tissue-engineered skin equivalents, and grafted to immunodeficient mice. As with normal and other genodermatosis-derived cells, skin regeneration occurred efficiently allowing the generation of a number of XP-C skin-engrafted animals. Engrafted animals maintained stable grafts up to 22 weeks, the longest time assessed. This result indicated that in vitro manipulation involving cell culture and skin bioengineering did not affect the regenerative capacity of $\mathrm{XP}-\mathrm{C}$ cells, which requires the presence and persistence of epidermal stem cells. Engrafted animals presented hyperpigmented skin (Figure 6C), a common feature seen also in XP-C patients (Figure 6A). Twelve weeks after grafting, mature XP-C skin regenerated on mice (Figure 6D) was histologically equivalent to that of donor skin biopsy showing no apparent phenotypic alterations (Figure 6B).

Once the model was established, we tested the acute responses to UVB and, considering the deficiency of XP keratinocytes in nucleotide excision repair, we concentrated on assessing the presumably functional inability of XP-C engrafted skin to remove the DNA-damaged cells as previously shown in vitro. ${ }^{26}$ Given the hyperpigmented nature of XP-C engrafted skins, we used age-matched African descent skin donor-derived engrafted mice as controls. Accordingly, a 4 BED UVB dose was required to achieve CPD induction. Kinetics of CPD induction and clearance were determined 2 hours and 72 hours after irradiation (Figure 7). Two hours after irradiation, both control and XP-C grafts showed abundant CPD-labeled nuclei distributed along different epidermal strata (Figure 7, A and C). Removal of damaged cells upwards (toward desquamation) was evident at 72 hours only in control (Figure 7B) skin but not in XP-C skins where CPD remained in all epidermal strata (Figure 7D), demonstrating the functional inability of human XP-C cells, for the first time, in vivo. This result, which confirms previous findings in a skin organotypic system, ${ }^{26}$ suggests the suitability of 
the XP-C skin-humanized model to test corrective approaches including ex vivo gene therapy.

\section{Discussion}

$\mathrm{UV}$ radiation is the main noxious and carcinogenic agent for human skin. Molecular changes associated to UVB irradiation have been extensively characterized in vitro in keratinocytes in culture. ${ }^{27,28}$ Although highly informative, these transcriptional profiling and other biochemical analyses are somewhat skewed by the fact that cultured keratinocytes represent only a mitotically activated basal cell compartment. The presence of differentiated cell layers of the epidermis achieved in 3D organotypic cultures allows for more accurate in vitro models to study UV effects. However, the organotypic systems often maintain a (hyper)proliferative basal stratum as compared with quiescent native human epidermis and only allow for relatively short-term studies. Reliable in vivo studies lack behind due to the ethical or practical constraints of using human volunteers or inaccurate animal models. Our group has developed a skin-humanized mouse platform based on skin tissue engineering and optimized surgical procedures in immunodeficient hosts enabling long-term human skin regeneration as well as skin disease modeling and therapeutic approaches. ${ }^{12}$

In this work we assessed the performance of our model in the acute UVB effects in vivo. By using Caucasian or African-descent donor keratinocytes we were able to regenerate differentially pigmented skins without the need of adding exogenous melanocytes to the system as in other tissue engineered-based models. ${ }^{29,30}$ In fact, our system relies on the persistence of resident melanocytes during short-term keratinocyte culture on lethally irradiated feeder layers. ${ }^{21}$ Under such conditions, cultures usually maintain their physiological 1:40 (melanocytes: keratinocytes) ratio, which is preserved also in graftable skin equivalents and regenerated skins. ${ }^{31,32}$ We were able to characterize dose-dependent nongenotoxic and DNA damaging UVB responses and their modulation by the degree of skin pigmentation. Our in vivo study confirms and extends previous results establishing a photoprotective effect of supranuclear melanin caps in native human skin explants. ${ }^{33}$ By using the appearance of sunburn cells as a histological marker of UVB genotoxic effects, we were also able to establish a nearly fourfold photoprotective factor of high melanin content as found for phototype VI skins. ${ }^{33,34}$ The model also proved satisfactory to test topic photoprotective agents as well as DNA damage repair kinetics after UVB irradiation. In fact, the physiological epidermal turnover and differentiation allowed the identification of CPD-containing cells in their transit to desquamation. While studies on the acute UVB effects in volunteers and human skin xenografts showed results similar to ours in terms of epidermal hyperplasia and keratin $\mathrm{K} 6$ induction, ${ }^{6,35}$ minor differences in the acanthotic response kinetics were noticed. It is likely that such discrepancies are the consequence of fine tuning variations in the MED/BED estimation in each particular experimental setting. In this regard, it is noteworthy that remarkable differences have been observed between 0.5 and $1 \mathrm{BED}$ in terms of functional epidermal cell proliferation kinetics.

In addition to the studies in normal regenerated human skin, a major goal was the establishment of an in vivo photosensitive skin humanized mouse model. That was achieved through the efficient engraftment of bioengineered skin equivalents composed of XP-C patient-derived keratinocytes and fibroblasts. The human XP-C regenerated skin allowed assessing the persistence of UVB-induced DNA damage for the first time in vivo. Our findings of CPD persistence recapitulated those previously described using XP-C organotypic skin cultures. ${ }^{26}$ However, in contrast to in vitro systems only reliable to assess acute short-lasting UVB effects, our XP-C model appears suitable to study chronic effects including mutagenesis and carcinogenesis, which require clonal expansion of damaged/initiated stem cells. Permanent skin regeneration involving stem cell preservation is also a requisite to test long-lasting therapeutic approaches such as gene therapy. As previously achieved using clinically relevant humanized in vivo models for dystrophic and junctional forms of Epidermolysis Bullosa, ${ }^{17,19}$ our new XP-C model certainly warrants testing ex vivo cutaneous gene therapy approaches for XP-C. Overall, our normal and XP-C skin-humanized models constitute valuable proxy tools (to avoid the use of human subjects) for research in the pathogenesis and therapeutics of UV-induced lesions.

\section{Acknowledgments}

We are indebted to Almudena Holguin and Blanca Duarte for excellent technical assistance and animal grafting. We thank Dr. Thierry Magnaldo for XPC patient diagnosis through retroviral complementation.

\section{References}

1. Mudgil AV, Segal N, Andriani F, Wang Y, Fusenig NE, Garlick JA: Ultraviolet B irradiation induces expansion of intraepithelial tumor cells in a tissue model of early cancer progression. J Invest Dermatol 2003, 121:191-197

2. Matsumura $\mathrm{Y}$, Ananthaswamy HN: Molecular mechanisms of photocarcinogenesis. Front Biosci 2002, 7:765-783

3. Kraemer KH: Sunlight and skin cancer: another link revealed. Proc Natl Acad Sci USA 1997, 94:11-14

4. Brash DE, Ziegler A, Jonason AS, Simon JA, Kunala S, Leffell DJ: Sunlight and sunburn in human skin cancer. p53, apoptosis, and tumor promotion. J Investig Dermatol Symp Proc 1996, 1:136-142

5. Setlow RB: The wavelengths in sunlight effective in producing skin cancer: a theoretical analysis. Proc Natl Acad Sci USA 1974, 71:3363-3366

6. Del Bino S, Vioux C, Rossio-Pasquier P, Jomard A, Demarchez M, Asselineau D, Bernerd F: Ultraviolet B induces hyperproliferation and modification of epidermal differentiation in normal human skin grafted on to nude mice. Br J Dermatol 2004, 150:658-667

7. Ruven HJ, Seelen CM, Lohman PH, van Kranen H, van Zeeland AA, Mullenders, LH: Strand-specific removal of cyclobutane pyrimidine dimers from the p53 gene in the epidermis of UVB-irradiated hairless mice. Oncogene 1994, 9:3427-3432

8. Laga AC, Murphy GF: The translational basis of human cutaneous photoaging: on models, methods, and meaning. Am J Pathol 2009, 174:357-360 
9. Soballe PW, Montone KT, Satyamoorthy K, Nesbit M, Herlyn M: Carcinogenesis in human skin grafted to SCID mice. Cancer Res 1996, 56:757-764

10. Atillasoy ES, Elenitsas R, Sauter ER, Soballe PW, Herlyn M: UVB induction of epithelial tumors in human skin using a RAG-1 mouse xenograft model. J Invest Dermatol 1997, 109:704-709

11. Berking C, Takemoto R, Binder RL, Hartman SM, Ruiter DJ, Gallagher PM, Lessin SR, Herlyn M: Photocarcinogenesis in human adult skin grafts. Carcinogenesis 2002, 23:181-187

12. Garcia M, Escamez MJ, Carretero M, Mirones I, Martinez-Santamaria L, Navarro M, Jorcano JL, Meana A, Del Rio M, Larcher F: Modeling normal and pathological processes through skin tissue engineering. Mol Carcinog 2007, 46:741-745

13. Llames S, Garcia E, Garcia V, Del Rio M, Larcher F, Jorcano JL, Lopez E, Holguin P, Miralles F, Otero J, Meana A: Clinical results of an autologous engineered skin. Cell Tissue Bank 2006, 7:47-53

14. Llames SG, Del Rio M, Larcher F, Garcia E, Garcia M, Escamez MJ, Jorcano JL, Holguin P, Meana A: Human plasma as a dermal scaffold for the generation of a completely autologous bioengineered skin. Transplantation 2004, 77:350-355

15. Escámez MJ, Carretero M, García M, Martínez-Santamaría L, Mirones I, Duarte B, Holguín A, García E, García V, Meana A, Jorcano JL, Larcher F, Del Río M: Assessment of optimal virus-mediated growth factor gene delivery for human cutaneous wound healing enhancement. J Invest Dermatol 2008, 128:1565-1575

16. Escamez MJ, Garcia M, Larcher F, Meana A, Munoz E, Jorcano JL, Del Rio M: An in vivo model of wound healing in genetically modified skin-humanized mice. J Invest Dermatol 2004, 123:1182-1191

17. Di Nunzio F, Maruggi G, Ferrari S, Di lorio E, Poletti V, Garcia M, Del Rio M, De Luca M, Larcher F, Pellegrini G, Mavilio F: Correction of laminin-5 deficiency in human epidermal stem cells by transcriptionally targeted lentiviral vectors. Mol Ther 2008, 16:1977-1985

18. Carretero M, Escamez MJ, Prada F, Mirones I, Garcia M, Holguin A, Duarte B, Podhajcer O, Jorcano JL, Larcher F, Del Rio M: Skin gene therapy for acquired and inherited disorders. Histol Histopathol 2006, 21:1233-1247

19. Gache Y, Baldeschi C, Del Rio M, Gagnoux-Palacios L, Larcher F, Lacour JP, Meneguzzi G: Construction of skin equivalents for gene therapy of recessive dystrophic epidermolysis bullosa. Hum Gene Ther 2004, 15:921-933

20. Arnaudeau-Bégard C, Brellier F, Chevallier-Lagente O, Hoeijmakers $J$, Bernerd F, Sarasin A, Magnaldo T: Genetic correction of DNA repair-deficient/cancer-prone xeroderma pigmentosum group $C$ keratinocytes. Hum Gene Ther 2003, 14:983-996

21. Rheinwald JG, Green H: Serial cultivation of strains of human epidermal keratinocytes: the formation of keratinizing colonies from single cells. Cell 1975, 6:331-343

22. Del Rio M, Larcher F, Serrano F, Meana A, Munoz M, Garcia M, Munoz E, Martin C, Bernad A, Jorcano JL: A preclinical model for the analysis of genetically modified human skin in vivo. Hum Gene Ther 2002, 13:959-968
23. Meana A, Iglesias J, Del Rio M, Larcher F, Madrigal B, Fresno MF, Martin C, San Roman F, Tevar F: Large surface of cultured human epithelium obtained on a dermal matrix based on live fibroblastcontaining fibrin gels. Burns 1998, 24:621-630

24. Bernerd F, Asselineau D: Successive alteration and recovery of epidermal differentiation and morphogenesis after specific UVB-damages in skin reconstructed in vitro. Dev Biol 1997, 183:123-138

25. Bernerd F, Vioux C, Lejeune F, Asselineau D: The sun protection factor (SPF) inadequately defines broad spectrum photoprotection: demonstration using skin reconstructed in vitro exposed to UVA. UVB or UV-solar simulated radiation. Eur J Dermatol 2003, 13:242-249

26. Bernerd F, Asselineau D, Vioux C, Chevallier-Lagente O, Bouadjar B, Sarasin A, Magnaldo T: Clues to epidermal cancer proneness revealed by reconstruction of DNA repair-deficient xeroderma pigmentosum skin in vitro. Proc Natl Acad Sci USA 2001, 98:7817-7822

27. Sesto A, Navarro M, Burslem F, Jorcano JL: Analysis of the ultraviolet $B$ response in primary human keratinocytes using oligonucleotide microarrays. Proc Natl Acad Sci USA 2002, 99:2965-2970

28. Li D, Turi TG, Schuck A, Freedberg IM, Khitrov G, Blumenberg M: Rays and arrays: the transcriptional program in the response of human epidermal keratinocytes to UVB illumination. FASEB J 2001, 15:2533-2535

29. Hachiya A, Sriwiriyanont P, Fujimura T, Ohuchi A, Kitahara T, Takema Y, Kitzmiller WJ, Visscher MO, Tsuboi R, Boissy RE: Mechanistic effects of long-term ultraviolet $B$ irradiation induce epidermal and dermal changes in human skin xenografts. Am J Pathol 2009, 174:401-413

30. Hachiya A, Sriwiriyanont P, Kaiho E, Kitahara T, Takema Y, Tsuboi R: An in vivo mouse model of human skin substitute containing spontaneously sorted melanocytes demonstrates physiological changes after UVB irradiation. J Invest Dermatol 2005, 125:364-372

31. Guerra L, Capurro S, Melchi F, Primavera G, Bondanza S, Cancedda R, Luci A, De Luca M, Pellegrini G: Treatment of "stable" vitiligo by Timedsurgery and transplantation of cultured epidermal autografts. Arch Dermatol 2000, 136:1380-1389

32. De Luca M, Franzi AT, D'Anna F, Zicca A, Albanese E, Bondanza S, Cancedda R: Coculture of human keratinocytes and melanocytes: differentiated melanocytes are physiologically organized in the basal layer of the cultured epithelium. Eur J Cell Biol 1988, 46:176-180

33. Kobayashi N, Nakagawa A, Muramatsu T, Yamashina $Y$, Shirai $T$, Hashimoto MW, Ishigaki Y, Ohnishi T, Mori T: Supranuclear melanin caps reduce ultraviolet-induced DNA photoproducts in human epidermis. J Invest Dermatol 1998, 110:806-810

34. Del Bino S, Sok J, Bessac E, Bernerd F: Relationship between skin response to ultraviolet exposure and skin color type. Pigment Cell Res 2006, 19:606-614

35. Lee JH, An HT, Chung JH, Kim KH, Eun HC, Cho KH: Acute effects of UVB radiation on the proliferation and differentiation of keratinocytes. Photodermatol Photoimmunol Photomed 2002, 18:253-261 